\title{
1 Analyzing the weak dimerization of a cellulose binding module by sedimentation velocity 2 experiments
}

4 Dmitrii Fedorov $^{1}$, Piotr Batys ${ }^{1,2}$, Maria Sammalkorpi ${ }^{1,3}$, and Markus B. Linder ${ }^{1 *}$

$5{ }^{1}$ Department of Bioproducts and Biosystems, School of Chemical Engineering, Aalto

6 University, Espoo, Finland

72 Jerzy Haber Institute of Catalysis and Surface Chemistry, Polish Academy of Sciences,

8 Krakow, Poland

$9{ }^{3}$ Department of Chemistry and Material Science, School of Chemical Engineering, Aalto

10 University, Espoo, Finland

11 *Corresponding author: E-mail: markus.linder@aalto.fi

\section{Abstract}

13 Cellulose binding modules (CBMs) are found widely in different proteins that act on cellulose.

14 Because they allow a very easy way of binding recombinant proteins to cellulose, they have

15 become widespread in many biotechnological applications involving cellulose. One commonly 16 used variant is the $\mathrm{CBM}_{\mathrm{CipA}}$ from Clostridium thermocellum. Here we studied the dimerization of

$17 \mathrm{CBM}_{\mathrm{CipA}}$, because we were interested if its solution behavior could have an impact on its use in

18 biotechnical applications. As the principal approach, we used sedimentation velocity analytical 19 ultracentrifugation. To enhance our understanding of the possible interactions, we used molecular 20 dynamics simulations. By analysis of the sedimentation velocity data using a discrete model 21 genetic algorithm we found that the $\mathrm{CBM}_{\mathrm{CipA}}$ shows a weak dimerization interaction with a 22 dissociation constant $K_{D}$ of about $87 \mu \mathrm{M}$. As the $K_{D}$ of CBM $_{\text {CipA }}$ binding to cellulose is about 0.6

$23 \mu \mathrm{M}$, we conclude that the dimerization is unlikely to affect cellulose binding. However, at the high 24 concentrations used in some applications of the $\mathrm{CMB}_{\mathrm{CipA}}$, its dimerization is likely to have an 25 effect on its solution behavior. The work shows that analytical ultracentrifugation is a very efficient 26 tool to analyze this type of weak interactions. Moreover, we provide here a protocol for data 27 analysis in the program Ultrascan for determining dissociation constants by sedimentation velocity 28 experiments. 


\section{Introduction}

31 Cellulose binding modules (CBMs) are proteins that are found as parts of many different enzymes

32 and other proteins that in one way or the other interact with cellulose. Their role is to bind to

33 cellulose and typically they do so by surface exposed aromatic side chains. It is well known that

34 CBMs play a crucial role in cellulose degradation and enzymatic activity (1). There are up to 40

35 different families of functionally related, but structurally different proteins that bind to

36 carbohydrates. They form an example of convergent evolution with different protein scaffolds

37 achieving a similar binding function (2). The details of these binding mechanisms differ between

38 the individual families, although they share some common elements such as aromatic-pyranose

39 ring stacking as part of the binding interface.

40 CBMs have been utilized in a large number of biotechnical applications because they provide an

41 easy way to control the binding of proteins to cellulose. Especially the ability to use recombinant

42 DNA technology to design new structurally engineered proteins with CBMs attached has led to a

43 wide range of applications. For example, the activity of enzymes can be enhanced by increasing

44 their binding to cellulose by CBMs. In one study, different types of CBMs were used to either

45 enhance or inhibit the activity of lytic polysaccharides monooxygenases (LPMO) by swapping

46 between different types of CBMs (3). The activity of hydrolases have also been increased by fusing

47 enzymes to CBMs (4). Immobilization to cellulose is also an application that has been explored.

48 CBMs can be used as a high-capacity purification tag, a targeting molecule or an affinity tag for

49 enzyme immobilization and processing (5). In addition, CBMs can be used to increase a rate of

50 catalysis by creating of association between biocatalysts and substrates $(6,7)$. Other applications

51 include; cell immobilization (8), diagnostics and even protective devices against nerve gas (9).

52 Because CBMs attach to cellulose surfaces, they can also be used to modify the materials 53 properties of cellulose based materials. An example of this was the improvement of the mechanical

54 properties of paper. A protein constructed to contain two CBMs at each end of a linker, resulted in 55 paper with higher folding endurance (10). In another example, a recombinant silk-protein based 56 adhesive for cellulose included CBMs (11).

57 Here we focus on the $\mathrm{CBM}_{\mathrm{CipA}}$ from the Cellulomonas fimi cellulosome anchoring protein CipA. 58 The $\mathrm{CBM}_{\mathrm{CipA}}$ belongs to family III according to the Cazy-classification (2). $\mathrm{CBM}_{\mathrm{CipA}}$ has a nine- 
59 stranded $\beta$ sandwich like structure with a jellyroll topology and belongs to surface binding type

60 of CBMs (type A). It has a face that contains planar linear strip of aromatic and polar residues,

61 which participates in the interaction with crystalline cellulose (12).

$62 \mathrm{CBM}_{\mathrm{CipA}}$ shows a very strong binding to crystalline cellulose, with a dissociation constant $\left(K_{D}\right)$ of 63 about $0.6 \mu \mathrm{M}(13-15)$. This is an order of magnitude stronger than the $K_{D}$ of, for example, the

64 fungal family I type of CBMs (16). Due to the high affinity of $\mathrm{CBM}_{\mathrm{CipA}}$ to cellulose, it has a high 65 practical value in applications. Because of its wide use and because any multimerization behavior possibly can influence the use of the protein in the applications, we decided to investigate the

67 multimerization characteristics of $\mathrm{CBM}_{\mathrm{CipA}}$ in more detail. Furthermore, a possibility of 68 dimerization was indicated in our previous work using $\mathrm{CBM}_{\mathrm{CipA}}$ as a fusion partner in silk-material 69 forming proteins $(11,17,18)$. For $\mathrm{CBM}_{\mathrm{CipA}}$, no detailed information of dimerization is available,

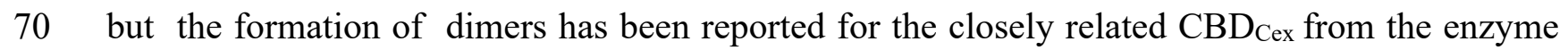
71 Cex, a $\beta$-1,4-glycanase from C.fimi (19). As a method to study the solution interactions of the $72 \mathrm{CBM}_{\mathrm{CipA}}$ we chose analytical ultracentrifugation (AUC) which is one of the most powerful 73 techniques available for quantifying weak interactions (20). Experiments performed by AUC are 74 either based on analyzing sedimentation velocity or sedimentation equilibrium. When performing 75 sedimentation velocity experiments, one obtains the dissociation constant $K_{D}$, the diffusion 76 coefficient, as well as information on the anisotropy of the protein in solution (21).

Materials and Methods

79 Protein expression. CBM CipA $_{\text {was }}$ codon optimized for E. coli and synthetized by GenScript USA 80 (NJ, USA), and cloned into the vector pET28a(+) as described earlier (15). The synthetized genes 81 contained BsaI restriction sites in their 5' and 3' ends. The multiple cloning site of the pET28a(+) 82 expression vector was modified to include two $B s a$ I restriction sites by inserting an adapter 83 constructed from oligonucleotides 5'-CATGGGGAGACCGCGGATCCGAATTCGGGTCTC-3' 84 and 5'-TCGAGAGACCCGAATTCGGATCCGCGGTCTCCC-3' into NcoI and XhoI restriction 85 sites. Plasmids were transformed to E. coli XL1-blue strain and plated on kanamycin $(50 \mu \mathrm{g} / \mathrm{mL})$ 86 containing LB-plates. The $\mathrm{CBM}_{\mathrm{CipA}}$ contained a 6-His-tag in the C-terminal end. The sequence of 87 the $\mathrm{CBM}_{\text {CipA }}$ was: 

AAIIGSNGSYNGITSNVKGTFVKMSSSTNNADTYLEISFTGGTLEPGAHVQIQGRFAKND WSNYTQSNDYSFKSASQFVEWDQVTAYLNGVLVWGKEPLEHHHHHH

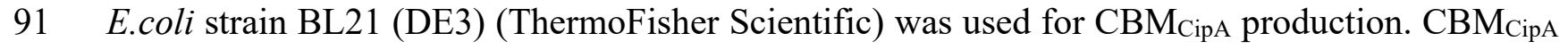
92 was expressed using MagicMedia ${ }^{\mathrm{TM}}$ (ThermoFisher Scientific) expression medium in accordance 93 with the manufacturers protocol. The purification process consisted of two parts, precipitation by

94 heating and by immobilized metal affinity chromatography (IMAC). The sample was heated up to $9570{ }^{\circ} \mathrm{C}$ for 30 minutes to precipitate impurities, and then clarified by centrifugation at $5000 \mathrm{rpm}$. 96 An ÄKTA-Pure liquid chromatography system (GE Healthcare Life Sciences) with a 5mL HisTrap 97 column was used for IMAC purification of the protein. The buffer was changed using desalting 98 columns.

99 Analytical ultracentrifugation. Sedimentation velocity experiments were performed using a 100 Beckman Coulter Optima AUC and UV-Vis absorbance optics at $280 \mathrm{~nm}$ wavelength. An An-50 101 rotor and measuring cells with $12 \mathrm{~mm}$ Epon centerpieces and quartz windows were used. Cleaning 102 of measuring cells was performed by deionized water with $13.0 \mathrm{M} \Omega \cdot \mathrm{cm}$ resistivity, $20 \%$ ethanol 103 and 1\% Hellmanex detergent. All measurements were carried out at $50000 \mathrm{rpm}$. Measurements 104 were made in phosphate-buffered saline $\left(137 \mathrm{mM} \mathrm{NaCl}, 2.7 \mathrm{mM} \mathrm{KCl}, 10 \mathrm{mM} \mathrm{Na}_{2} \mathrm{HPO}_{4}\right.$, and 1.8 $\left.105 \mathrm{mM} \mathrm{KH}_{2} \mathrm{PO}_{4}, \mathrm{pH} 7.35\right)$. $\mathrm{CBM}$ samples were measured in the concentration range from $0.05 \mathrm{~g} / \mathrm{L}$ to $106 \quad 0.7 \mathrm{~g} / \mathrm{L}$ that corresponds to $0.1-1.3 \mathrm{OD}$ range. $400 \mu \mathrm{L}$ of sample was loaded in each measuring 107 cell. All experiments were performed at $20{ }^{\circ} \mathrm{C}$ and prior to each experiment the system was 108 thermally stabilized for $1 \mathrm{~h} 30 \mathrm{~min}$. To get complete sedimentation and sufficient amounts of data 109 points, 600 scans per each sample were collected with a frequency of one scan per 80 seconds.

110 Data analysis by Ultrascan. Data analysis were performed using the software UltraScan III (v. 4.0 111 revision 2466) (22). Rotor-stretching calibration and chromatic aberration corrections were applied 112 during data import. A partial specific volume $(\bar{v})$ of $0.7148 \mathrm{~mL} / \mathrm{g}$ was used. It was based on the 113 amino acid sequence and calculations by the estimation tool implemented in Ultrascan. A 114 theoretical density of $1.0056 \mathrm{~g} / \mathrm{mL}$ and viscosity of $1.0120 \mathrm{mPa} \cdot \mathrm{s}$ for the buffer were calculated 115 using Ultrascan. During data editing, the meniscus position and end cutoff distance were entered 116 manually. The plateau position corresponding to a uniform concentration prior to acceleration and 117 the baseline buffer absorbance were determined automatically. 
118 Concentration profiles obtained in the sedimentation velocity experiments were treated using 2119 dimensional spectrum analysis (2DSA) (23). We used 2DSA to perform primary analysis coupled 120 with simultaneous time-invariant and radial-invariant noise reduction to obtain the frictional ratio $121\left(f / f_{0}\right)$, and sedimentation coefficient $(s)$ values. The grid parameters used were 1 to 10 for $s$ and 1 122 to 4 for $\mathrm{f} / \mathrm{f}_{0}$ with 64 grid points in each direction. The choice of parameters was based on time123 derivate analysis, and was selected to cover the distribution of parameters for all species in 124 solution. The 2DSA was done in three steps. First, the time-invariant noise was removed after 125 which the meniscus was fitted. The meniscus fit range was $0.03 \mathrm{~cm}$ around the previously chosen 126 position. In the meniscus fitting procedure, 10 positions were chosen and corresponding root mean 127 square deviation (RMSD) values were approximated by second order polynomial to determine the 128 position with lowest RMSD. The position with lowest RMSD was used for further analysis. The 129 third step was an iterative 2DSA refinement consisting of repetitions with improved meniscus 130 position and fitting of the noise profile. The number of iterations was 10, which was sufficient for 131 convergence of the fit. The parameters of the grid were the same in all steps.

132 The 2DSA results and noise reduction profile obtained by the analysis described above were used 133 for further analysis. A genetic algorithm (GA) and a genetic algorithm Monte Carlo (GAMC) (24) 134 optimization were used to extract the main populations of frictional ratio and sedimentation 135 coefficient combinations present in the data. The existence of a reversible self-association was 136 demonstrated using a van Holde-Weischet (vHW) (25) analysis. The dissociation constant $\left(K_{D}\right)$ 137 was determined by a discrete model genetic algorithm (DMGA). The specific parameters used in 138 GA, GAMC, vHW and DMGA are provided in the supplemental information. Hydrodynamic 139 parameters of monomer and dimer were estimated using the UltraScan Solution Modeler (US140 SOMO) (26). Calculations were performed using the ZENO hydrodynamic computation algorithm 141 (27-29) and the van der Waals overlap bead model (30).

142 Molecular modelling. All-atom molecular dynamics (MD) simulations were carried out by using 143 the Gromacs software (version 5.1.4) (31,32). We used the particle Mesh Ewald (PME) 144 electrostatics calculation scheme (33), and a non-bonded interactions cut-off of $1 \mathrm{~nm}$. The 145 modeling was performed with the Amber03ws force field (34) and TIP4P2005 water model (35).

146 Two systems were studied, one with a single $\mathrm{CBM}_{\mathrm{CipA}}$ molecule and one with two CBM $\mathrm{CipA}_{\mathrm{A}}$ 147 molecules in the simulation box. A cubic box of $8 \mathrm{~nm} \times 8 \mathrm{~nm} \times 8 \mathrm{~nm}$ in initial size was used for 
148 the single $\mathrm{CBM}_{\mathrm{CipA}}$ system and for the two molecule-system, a box size of $12.5 \mathrm{~nm} \times 12.5 \mathrm{~nm} \times$

$14912.5 \mathrm{~nm}$ was used (Fig 1).

150 The initial crystal structure of the single $\mathrm{CBM}_{\mathrm{CipA}}$ simulations was the Protein Data Bank structure 151 1NBC. The file was modified to leave only the protein itself. The amino acid sequence differs 152 from the sequence of the real protein by a missing polyHis-tag and two amino acids in the 153 beginning and the end of the sequence. The initial structure for the two-molecule system was 154 obtained using the final frame of the single CBM simulation, cutting the protein together with a $1551 \mathrm{~nm}$ water shell. The initial arrangement of the two molecules relative to each other was chosen 156 based on the location of the $\beta$-sheets and the geometric shape. After this, the proteins were solvated 157 with explicit water molecules. Neutralization of the net charge in the system was performed by 158 replacement of water molecules by $\mathrm{Na}^{+}$ions: 4 ions were added in the two-molecule case. Both 159 systems were energy minimized. After initial equilibration, the simulations were performed in the 160 NPT (isothermal-isobaric) ensemble. The temperature and pressure control were carried out by the 161 V-rescale thermostat (36) with a time constant of 0.1 ps and the Parrinello-Rahman barostat (37) 162 with a time constant of $2 \mathrm{ps}$. The temperature and pressure reference levels were set at $300 \mathrm{~K}$ and 1631 bar. Visualization was done with the VMD software (v. 1.9.4a9) (38). The main simulation was 164 conducted for $160 \mathrm{~ns}$ for the single $\mathrm{CBM}_{\mathrm{CipA}}$ and for $450 \mathrm{~ns}$ for the two-molecule system. In the 165 two-molecule simulation, the first $150 \mathrm{~ns}$ were disregarded in the analysis. 


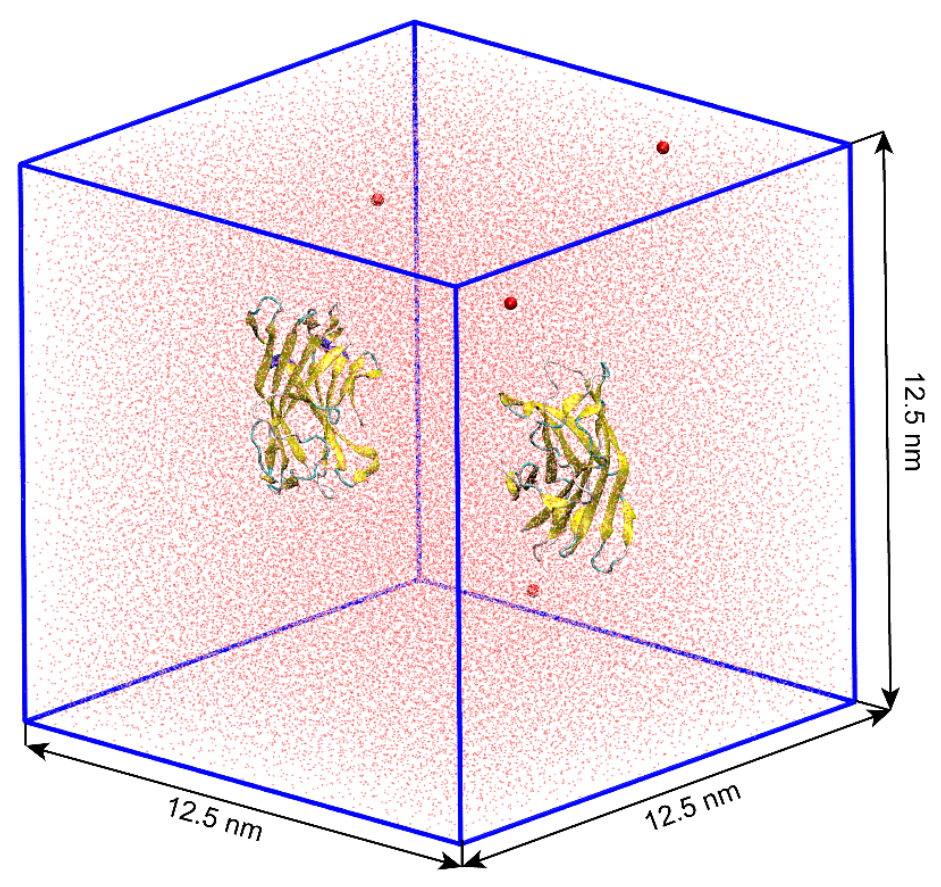

168 Fig. 1. The initial all-atom molecular dynamics simulation configuration of two CBM CipA molecules in the simulation box. The explicit water molecules are shown as red dots and the $\mathrm{Na}^{+}$ ions that are required for charge neutrality as red spheres.

171 Diffusion coefficients were calculated using the mean square displacement (MSD). The stable 172 linear region on the MSD plot was approximated by a straight line according to the equation

$$
\left\langle|r(t)-r(0)|^{2}\right\rangle=6 D t
$$

174 where $\left\langle|r(t)-r(0)|^{2}\right\rangle$ is the MSD, $D$ is the diffusion coefficient and $t$ is the time.

\section{Results and Discussion}

177 Sedimentation velocity experiments were conducted to investigate the possible oligomerization of

$178 \mathrm{CBM}_{\mathrm{CipA}}$ molecules. A set of solutions of $\mathrm{CBM}_{\mathrm{CipA}}$ at different concentration were studied to 179 follow changes in composition. After initial data treatment and noise reduction by 2DSA, GA was 180 used further data refinement. An example of a sedimentation profile and fitted data by the GA at $1810.7 \mathrm{~g} / \mathrm{L}$ concentration is shown in Fig $2 \mathrm{a}$. The GA analysis revealed two clearly identifiable peaks 182 at $2.15 \mathrm{~Sv}$ and $2.7 \mathrm{~Sv}$ (Fig 2b, Table 1). These peaks correspond to molecular weights of 18 and $18337 \mathrm{kDa}$. The $18 \mathrm{kDa}$ peak at $2.15 \mathrm{~Sv}$ corresponds to the calculated molecular weight of CBM $\mathrm{CipA}_{\mathrm{P}}$ 
184 as a monomer which is $18469 \mathrm{Da}$. The peak at 2.7 Sv corresponds very closely to the mass of

$185 \mathrm{CBM}_{\mathrm{CipA}}$ as a dimer which is $36938 \mathrm{Da}$. In addition, there was low intensity signal at $3.5 \mathrm{~Sv}$ that

186 could represent minor amounts of higher oligomerization states but which is more likely to be an

187 artefact due to random noise or sample impurities, as it did not show a concentration dependency.

188 We conclude that the data show the presence of monomer-dimer system in which monomeric state 189 prevails and is in good agreement of experimental and fitted data, and having a low overall level 190 of noise.

191
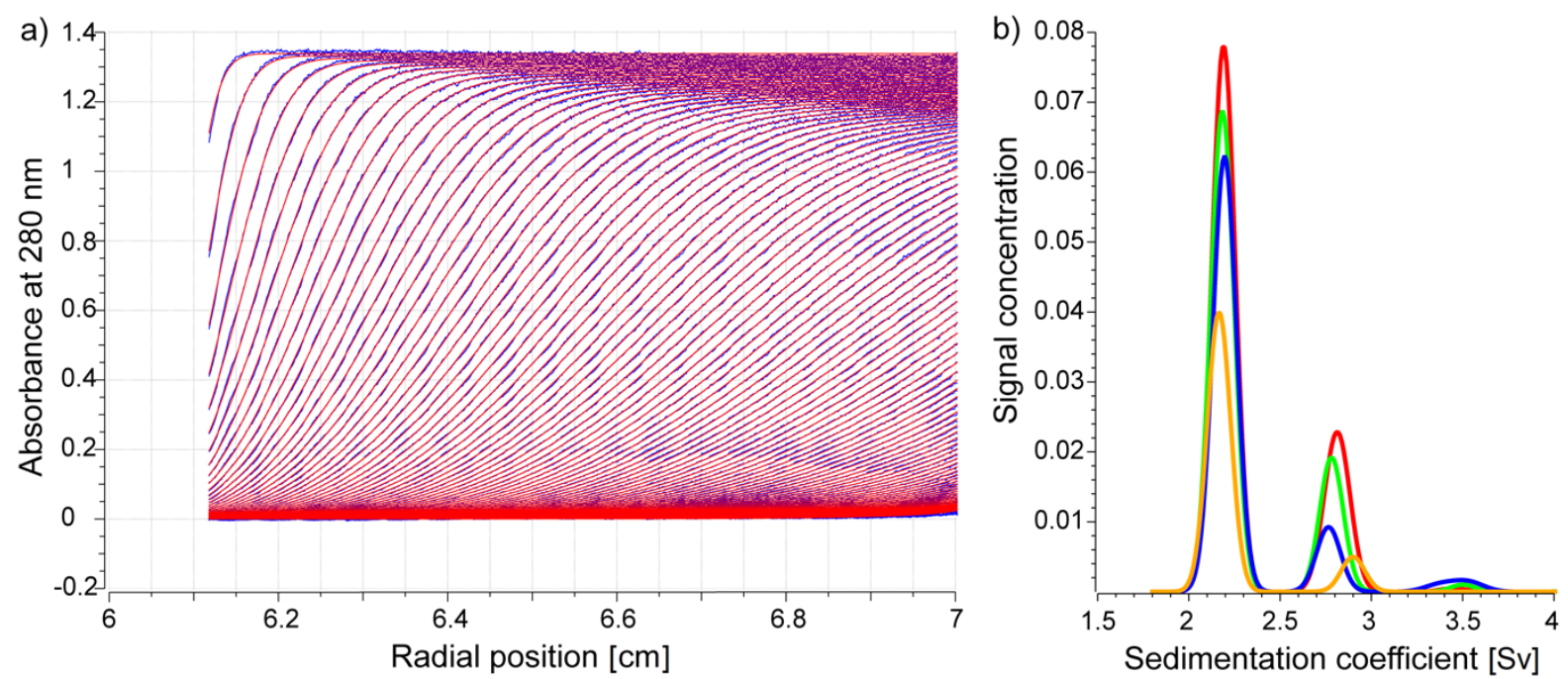

193 Fig. 2. a) Sedimentation velocity experimental data (blue lines) and fitted curves based on the GA

194 (red lines). b) GA analysis result shown as sedimentation distribution plot at four concentrations:

$1950.3 \mathrm{~g} / \mathrm{L}$ - yellow, $0.5 \mathrm{~g} / \mathrm{L}-$ blue, $0,6 \mathrm{~g} / \mathrm{L}$ - green and $0,7 \mathrm{~g} / \mathrm{L}$ red lines.

197 We conducted a GAMC analysis to evaluate the quality of data treatment and to perform 198 confidence analysis by statistical methods. The pseudo 3Ds distribution connects sedimentation 199 coefficients $(s)$, frictional ratios $\left(f / f_{0}\right)$, and partial concentrations of the solution. The peak at $3.5 \mathrm{~Sv}$ 200 had a very low intensity and was not been taken for further analysis. No significant changes were 201 observed in the pseudo 3D distribution after 64 Monte Carlo iterations (Fig 3). The analysis 202 showed excellent stability and it allows us to conclude that both peaks were determined in correct 203 way and definitely represent the solution composition. 


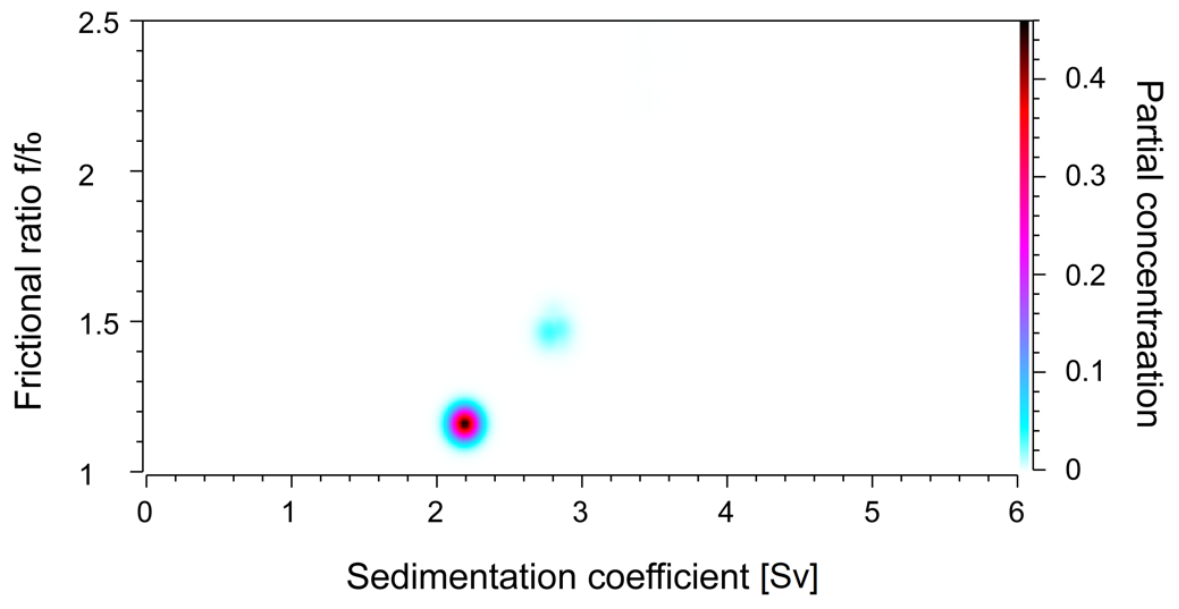

205 Fig. 3 Pseudo 3D plot of the CBM CipA solution composition at a concentration of $0.7 \mathrm{~g} / \mathrm{l}$ after 64

206 Monte Carlo iterations by GAMC. The graph shows two peaks, a monomer at $2.2 \mathrm{~Sv}$ and $207 f / f_{0} \approx 1.15$ and a dimer at $2.8 \mathrm{~Sv}$ and $f / f_{0} \approx 1.5$.

209 GAMC was then used to determine the diffusion constant $(D), s, f / f_{0}$, and partial concentration 210 values of the monomers and dimers for the full range of concentrations, i.e. from 0.05 to $0.7 \mathrm{~g} / 1$ 211 (Table 1). The sedimentation velocity fitting showed low RMSD values. The fitting was overall in 212 good agreement with sedimentation data having only small changes in parameters at different 213 concentrations. The CBM $\mathrm{CipA}$ monomer showed an average $s$ of $2.16 \mathrm{~Sv}$ and a $D$ of $10.5 \cdot 10^{-7} \mathrm{~cm}^{2} / \mathrm{s}$. 214 The $f / f_{0}$ of the monomer was equal to 1.2 indicating that the monomer has compact globular 215 structure as expected based on crystal structure. The dimer also showed expected values: a higher $216 s$ value of $2.8 \mathrm{~Sv}, D=6 \cdot 10^{-7} \mathrm{~cm}^{2} / \mathrm{s}$ and a $f / f_{0}$ of 1.6 , representing a more anisotropic form. The 217 partial concentrations of the two components showed a change with the total concentration, 218 indicating the presence of dynamic interactions. In other words, the $\mathrm{CBM}_{\mathrm{CipA}}$ behaved as 219 reversible system. This is still further evidence that the peak at $2.7 \mathrm{~Sv}$ represents a dimeric form. 
Table 1. Sedimentation velocity fitting results for 7 different loading concentrations. Parameters

224 of monomer and dimer peaks and also RMSD of the fitting are shown. Data for the dimer peak is

225 shown only for the concentrations where the peak was clearly identifiable.

\begin{tabular}{|c|c|c|c|c|c|c|c|c|c|}
\hline & Mon & mer frac & ion & & Dime & fraction & & & \\
\hline $\begin{array}{l}\text { Conc. } \\
{[\mathrm{mg} / \mathrm{mL}]}\end{array}$ & $\begin{array}{l}s \\
{[\mathrm{~Sv}]}\end{array}$ & $\begin{array}{l}D \cdot 10^{7} \\
{\left[\mathrm{~cm}^{2} / \mathrm{s}\right]}\end{array}$ & $f / f_{0}$ & $\begin{array}{l}\text { partial } \\
\text { conc. [\%] }\end{array}$ & $\begin{array}{l}s \\
{[\mathrm{~Sv}]}\end{array}$ & $\begin{array}{l}D \cdot 10^{7} \\
{\left[\mathrm{~cm}^{2} / \mathrm{s}\right]}\end{array}$ & $f / f_{0}$ & $\begin{array}{l}\text { partial } \\
\text { conc. } \\
{[\%]}\end{array}$ & $\begin{array}{l}\text { RMSD } \\
\text { of data } \\
\text { fitting }\end{array}$ \\
\hline 0.05 & 2.09 & 9.8 & 1.26 & 95.4 & & & & & 0.00086 \\
\hline 0.1 & 2.13 & 10.4 & 1.21 & 95.6 & & & & & 0.00102 \\
\hline 0.2 & 2.15 & 10.4 & 1.19 & 91.4 & & & & & 0.00119 \\
\hline 0.3 & 2.17 & 10.6 & 1.16 & 88.5 & 2.90 & 5.5 & 1.68 & 11.0 & 0.00144 \\
\hline 0.5 & 2.19 & 11.0 & 1.16 & 82.9 & 2.76 & 5.3 & 1.74 & 12.6 & 0.00209 \\
\hline 0.6 & 2.18 & 10.9 & 1.16 & 76.8 & 2.78 & 6.9 & 1.46 & 21.6 & 0.00248 \\
\hline 0.7 & 2.19 & 10.9 & 1.16 & 74.7 & 2.81 & 6.8 & 1.47 & 24.0 & 0.00269 \\
\hline Average & 2.16 & 10.5 & 1.2 & & 2.8 & 6.0 & 1.6 & & \\
\hline
\end{tabular}

229 We used the vHW analysis as an alternative way to the GAMC analysis to investigate the occurrence of reversible oligomerization. The vHW method is based on a graphical transformation

231 of the sedimentation velocity experimental data and allows obtaining a sedimentation coefficient

232 distribution that is independent of diffusion. By the vHW method, one can identify boundary 233 spreading due to diffusion and heterogeneity in the sedimentation coefficient as well as 234 differentiation between non-interacting and self-associating species. If the kinetics are slow 235 compared to the time of the experiment, the interacting components will be separated by the 236 centrifugal force and the boundary of concentration profile will be separated. However, in the case 
237 of relatively fast kinetics the components will have time to re-equilibrate and a single boundary is 238 observed. Changing the sample concentration will change the ratio between species causing 239 changes in the boundary shape. This change represents a shift toward higher sedimentation 240 coefficient and higher partial concentration of oligomers and can be seen in the vHW integral 241 distribution. Such a shift in the vHW distribution at different concentrations was observed for $242 \mathrm{CBM}_{\mathrm{CipA}}$ (Fig 4). The presence of the shift clearly indicates reversible self-association (21). Using 243 DMGA we calculated the dissociation constant $\left(K_{D}\right)$ values for this reversible self-association 244 (Table 2).

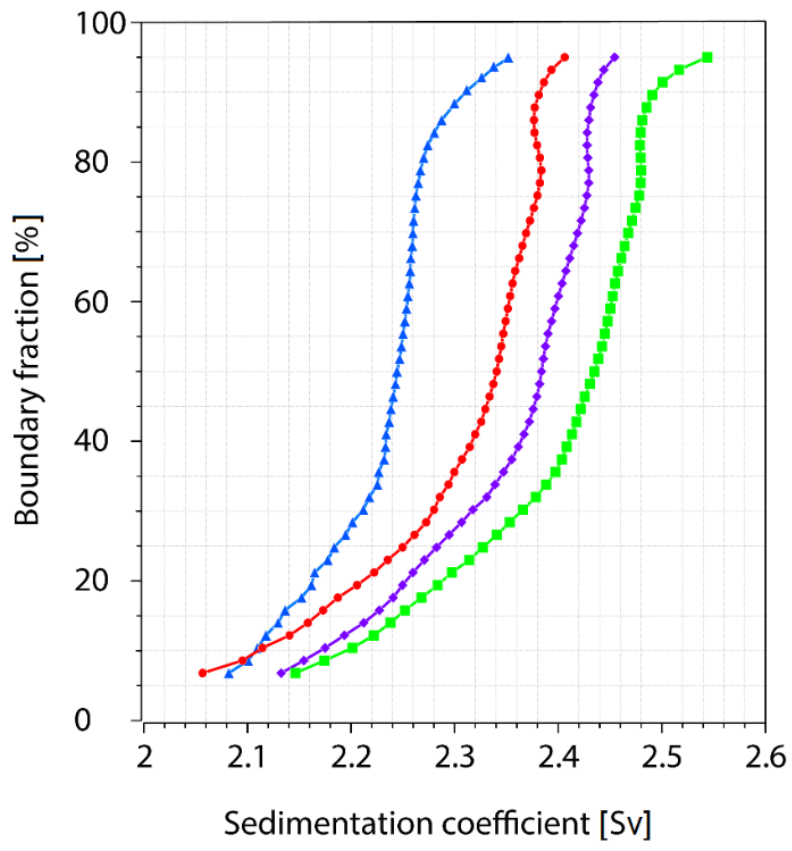

246 Fig. 4. A van Holde-Weischet integral distribution plot for four different loading concentrations:

$2470.3 \mathrm{~g} / \mathrm{L}$ - blue triangles, $0.5 \mathrm{~g} / \mathrm{L}$ - red circles, $0.6 \mathrm{~g} / \mathrm{L}$ - violet diamonds and $0.7 \mathrm{~g} / \mathrm{L}$ - green 248 squares. A clear shift of the curves towards higher Sv values correspond to a reversible self249 association of the components. 
Table 2. The value of the dissociation constant $K_{D}$ as determined at different concentrations.

\begin{tabular}{|l|l|l|l|}
\hline & & \multicolumn{2}{|l|}{ in\% confidence } \\
\hline Conc. $[\mathrm{mg} / \mathrm{mL}]([\mu \mathrm{M}])$ & $K_{D},[\mu \mathrm{M}]$ & lower & upper \\
\hline $0.20(10.83)$ & 81.3 & 68.8 & 93.8 \\
$0.30(16.24)$ & 86.7 & 76.9 & 94.5 \\
$0.50(27.07)$ & 93.9 & 81.3 & 106.6 \\
$0.60(32.49)$ & 92.3 & 83.4 & 101.1 \\
$0.70(37.90)$ & 80.5 & 68.8 & 92.2 \\
\hline Average & 87.0 & & \\
\hline
\end{tabular}

258 Independently from the sedimentation velocity experiments, molecular dynamics simulations were

259 also conducted. Two types of systems were simulated: one with a single $\mathrm{CBM}_{\mathrm{CipA}}$ molecule and 260 one system with two $\mathrm{CBM}_{\mathrm{CipA}}$ molecules in the box. The first system was used to estimate the 261 hydrodynamic properties of the $\mathrm{CBM}_{\mathrm{CipA}}$ molecule as a monomer. From this, a diffusion 262 coefficient of $7.9 \cdot 10^{-7} \mathrm{~cm}^{2} / \mathrm{s}$ was calculated. Compared to the average measured $D$ of $10.5 \cdot 10^{-7}$ $263 \mathrm{~cm}^{2} / \mathrm{s}$, we consider the agreement good considering the widely different approaches used. One 264 should note, however, that the MD simulations of the single $\mathrm{CBM}_{\mathrm{CipA}}$ refer to the dilute solution 265 in experiments as protein-protein interactions in the single CBM system would be interactions with 266 periodic images via the periodic boundary conditions. Indeed, the monomer $D$ decreases with the 267 concentration (Table 1) and at the lowest concentration, i.e., $0.05 \mathrm{~g} / \mathrm{L}$, the $D$ agrees slightly better 268 with the MD results.

269 The second system allowed us to study the process of CBM dimerization. Initially, the CBMs were 270 apart in the simulation box (Fig 1) but a stable dimer form was observed after $150 \mathrm{~ns}$ of MD 271 simulations and the dimer form stayed the same until the end of simulation, i.e. next $300 \mathrm{~ns}$ (Fig 272 5). The $\mathrm{CBM}_{\mathrm{CipA}}$ dimer diffusion coefficient was determined to be $6.2 \cdot 10^{-7} \mathrm{~cm}^{2} / \mathrm{s}$, which is in very 
273 good agreement with the measured one of $6.0 \cdot 10^{-7} \mathrm{~cm}^{2} / \mathrm{s}$. While the single monomer in the

274 simulation box corresponds to concentration $6.0 \mathrm{~g} / \mathrm{L}$, the two CBMs in a simulation box

275 correspond to concentration $3.1 \mathrm{~g} / \mathrm{L}$. The latter concentration is significantly above the

276 concentration range studied in the experiments. This suggests, that the $D$ determined from the MD

277 simulations should actually be compared with the $0.7 \mathrm{~g} / \mathrm{L}$ diffusion coefficient value, i.e. $6.8 \cdot 10^{-7}$

$278 \mathrm{~cm}^{2} / \mathrm{s}$ and not the dilute solution value.

279 For both the monomer and the dimer, the $D$ calculated from the MD simulations is underestimated

280 by $12-20 \%$. This might originate from the oversimplifications in the modelling methodology. For

281 example, the $D$ of the TIP4P/2005 water which is used in this work, is $\sim 10 \%$ smaller than

282 experimental value (35). However, the MD method properly catches the decrease in $D$ caused by

283 dimerization.

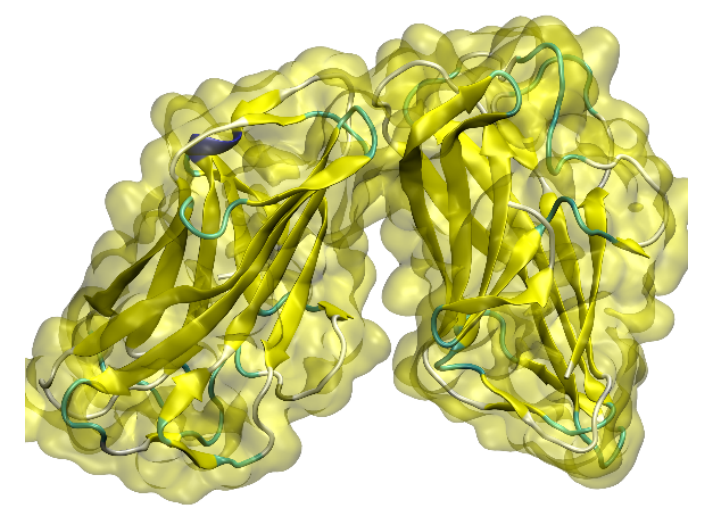

286 Fig. 5. CBM CipA dimer structure as obtained by the MD simulations. The snapshot corresponds to 287 the final configuration at $450 \mathrm{~ns}$.

288 A residue contact map (RCM) (Fig 6) was calculated from the two CBM simulation trajectory to 289 understand which residues are involved in the dimer formation in the simulated system. The RCM 290 shows the mean distance between the CBM residues, that is, each point on the map shows the 291 distance between two residues, as specified by the residue index. We plotted the two CBM 292 molecules with consecutive numbering, meaning that the lower left quadrant shows interactions 293 within molecule 1, the upper right quadrant shows interactions within molecule 2, and both the 
294 lower right and upper left show interactions between molecules. Notably, an RCM is symmetric

295 with respect to the diagonal from lower left to upper right. Residues on this line have a distance

296 equal to zero, since each residue on this line is compared to itself. Square areas through which the

297 zero-line passes display the distances between residues inside the molecule. The lines parallel and

298 perpendicular to the zero line inside these areas identify parallel and anti-parallel $\beta$-sheets. The

299 distance between residues belonging to different molecules is shown in square areas perpendicular

300 to the zero line. The RCM revealed at least two areas which can participate in dimer formation and

301 are involved in interactions between CBMs. The first area represents the interaction between

302 residues Met-81 and Ser-82 of one CBM and Gly-65 of the other CBM. The second area shows

303 interaction between Gln-48 of first molecule and group of residues Ser-63, Glu-102, Pro-103, Ala-

304105 belonging to the second molecule.

305
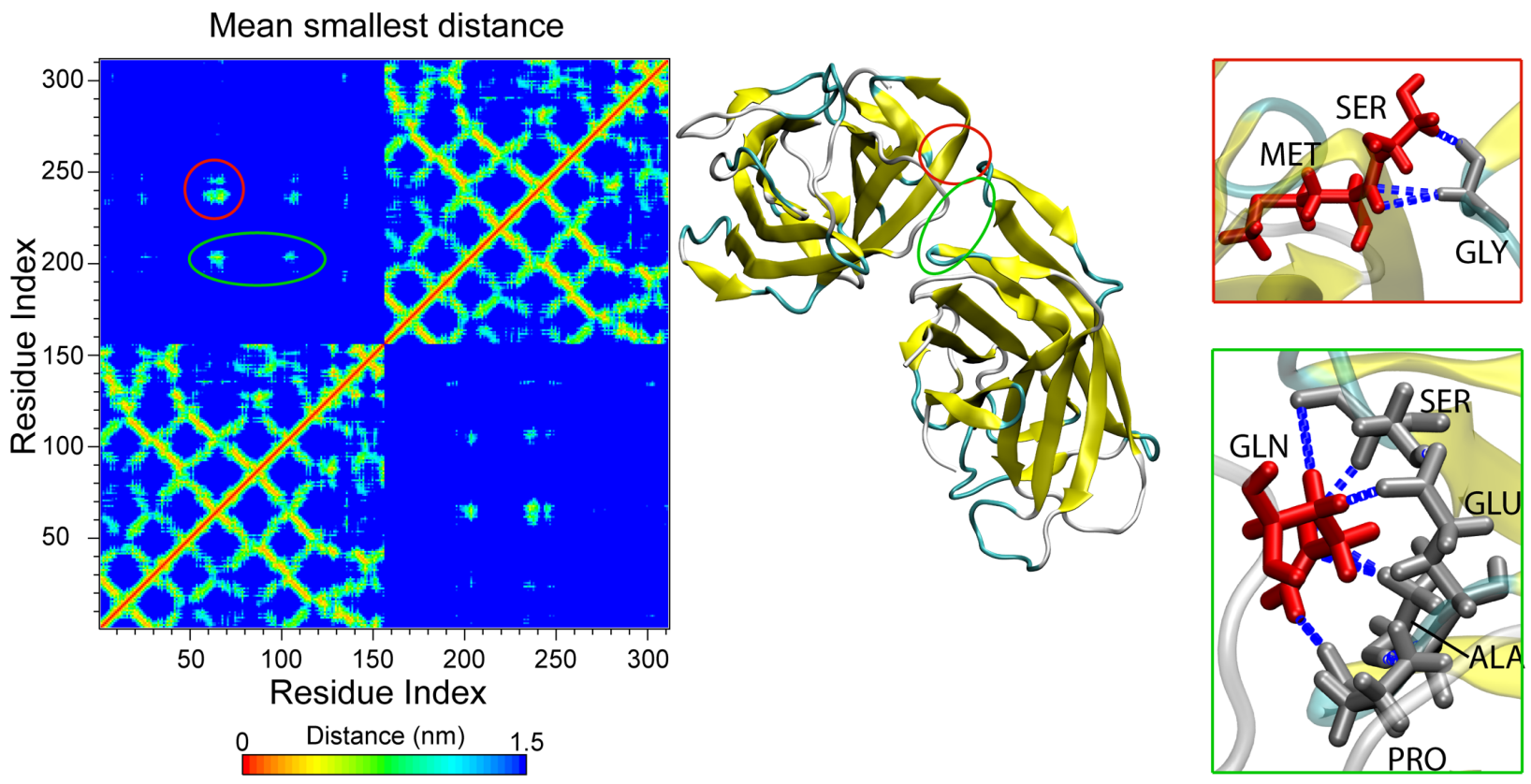

307 Fig. 6. Residue contact map calculated for the two CBM molecular dynamics simulations and the

308 corresponding dimer configuration with contact regions zoomed in. Red and green circles show

309 areas of contact between molecules. Hydrogen bonds are shown by blue dot lines.

311 We also used SOMO to estimate the results of the sedimentation velocity experiments. The

312 SOMO-estimations were based on the crystal structure for the monomer and the molecular 
313 dynamic simulations for the dimer. Table 3 shows that experimental and predicted values are in

314 good agreement for the monomer but were slightly off for the dimer. Both sets of SOMO data

315 show overestimation of $s$ and $D$ and underestimation of the $f / f_{0}$. The slight difference between

316 experimental and SOMO results could be explained by the absence of a poly-His tag and a few

317 other amino acids in the crystal structure. In the case of the dimer, an inexact partial specific

318 volume $(\bar{v})$ could also contribute.

Table 3. Comparison between experimental results at concentration $0.7 \mathrm{~g} / \mathrm{l}$ and SOMO modelling.

\begin{tabular}{|l|l|l|l|l|}
\hline & \multicolumn{3}{|l|}{ Monomer } \\
\hline & $s[\mathrm{~Sv}]$ & $\begin{array}{l}D \cdot 10^{7} \\
{\left[\mathrm{~cm}^{2} / \mathrm{s}\right]}\end{array}$ & $f / f_{0}$ & $\begin{array}{l}\bar{v} \\
{[\mathrm{~mL} / \mathrm{g}]}\end{array}$ \\
\hline Experimental & 2.19 & 10.9 & 1.16 & 0.7148 \\
\hline SOMO & 2.23 & 11.1 & 1.13 & 0.7180 \\
\hline & Dimer & & & \\
\hline Experimental & 2.81 & 6.8 & 1.47 & 0.7148 \\
\hline SOMO & 3.08 & 8.0 & 1.26 & 0.71 \\
\hline
\end{tabular}

\section{Conclusions}

326 We showed that the $\mathrm{CBM}_{\mathrm{CipA}}$ form dimers with a weak $K_{D}$ of approximately $87 \mu \mathrm{M}$. The 327 interaction can be classified as weak, on the edge of ultra-weak, as typically interactions that have 328 a $K_{D}$ over $100 \mu \mathrm{M}$ are classified as ultra-weak (20). The $K_{D}$ corresponds to a half of the CBM $\mathrm{CipA}_{\mathrm{C}}$ 329 molecules being as dimers at a concentration of $1.6 \mathrm{~g} / \mathrm{L}$. As the $K_{D}$ for the binding of $\mathrm{CBM}_{\mathrm{CipA}}$ is 330 at around $0.6 \mu \mathrm{M}(13-15)$, which is around two orders of magnitude lower we conclude that its 
331 dimerization has a negligible effect on any function involving cellulose binding. However, in other

332 uses of the CBM such as using then as part of molecular adhesives, much higher concentrations

333 were used reaching over $2 \mathrm{mM}(11,17)$. At these concentrations the majority of protein molecules

334 are in the dimer form, unless the $K_{D}$ is affected when the $\mathrm{CBM}_{\mathrm{CipA}}$ is part of a fusion protein.

335 Modelling indicated a possible mechanism of interaction between CBM molecules, and the

336 modelled structures showed calculated properties such as diffusion and frictional ratio that had

337 good agreement with the experimentally determined ones. In general AUC is suitable for studying

338 weak and ultraweak interactions, and several examples are found. Approaches include both

339 sedimentation velocity and sedimentation equilibrium setups and use of different software-

340 packages for analysis (39-42). Meanwhile, the software capabilities are constantly developing and

341 we found that the relatively recent approach of using the discrete model genetic algorithm

342 (DMGA) method to determine the $K_{D}$ to be a very efficient tool.

\section{Acknowledgements}

347 We thank the Aalto School of Chemical Engineering graduate school for funding (DF). This work 348 was supported by the Academy of Finland through its Centres of Excellence Programme (2014349 2019, HYBER) and under Projects No. 307474 and 309324 (MS). We are grateful for the support

350 by the FinnCERES Materials Bioeconomy Ecosystem and the Bioeconomy Infrastructure at Aalto.

351 Computational resources by CSC IT Centre for Science and RAMI - RawMatTERS Finland 352 Infrastructure are also gratefully acknowledged. Dr Sanni Voutilainen is acknowledged for cloning 353 the $\mathrm{CBM}_{\mathrm{CipA}}$. We thank professor Borries Demeler for his invaluable help with setting up 354 Ultrascan. 


\section{References}

1. Shoseyov O, Shani Z, Levy I. Carbohydrate Binding Modules : Biochemical Properties and Novel Applications. 2006;70(2):283-95.

2. The CAZypediaConsortium. Ten years of CAZypedia: a living encyclopedia of carbohydrate-active enzymes. Glycobiology. 2017;28:3-8.

3. Crouch LI, Labourel A, Walton PH, Davies GJ, Gilbert HJ. The contribution of non-catalytic carbohydrate binding modules to the activity of lytic polysaccharide monooxygenases. J Biol Chem. 2016;291(14):7439-49.

4. Kim H, Goto M, Jeong H, Jung KHWA, Kwon II, Furukawa K. Functional Analysis of a Hybrid Endoglucanase of Bacterial Origin Having a Cellulose Binding Domain from a Fungal Exoglucanase. Appl Biochem Biotechnol. 1998;

5. Boraston, A. B., B. W. McLean, J. M. Kormos, M. Alam, N. R. Gilkes CA, Haynes, P. Tomme, D. G.

6. Srisodsuk M, Lehtiö J, Linder M, Margolles-Clark E, Reinikainen T, Teeri TT. Trichoderma reesei cellobiohydrolase I with an endoglucanase cellulose-binding domain: Action on bacterial microcrystalline cellulose. J Biotechnol. 1997;57(1-3).

7. Limo MC, Penttila M. Addition of substrate-binding domains increases substrate-binding capacity and specific activity of a chitinase from Trichoderma harzianum. 2001;198:57-63.

8. Wang AA, Mulchandani A, Chen W. Whole-Cell Immobilization Using Cell Surface-Exposed

9. Wang AA, Mulchandani A, Chen W. Specific Adhesion to Cellulose and Hydrolysis of Surface-Expressed Cellulose-Binding Domain and Organophosphorus Hydrolase. 
http://ojs.cnr.ncsu.edu/index.php/BioRes/article/view/5048

11. Mohammadi P, Beaune G, Stokke BT, Timonen JVI, Linder MB. Self-Coacervation of a Silk-Like Protein and Its Use As an Adhesive for Cellulosic Materials. ACS Macro Lett. 2018;7:1120-5.

12. Tormo J, Lamed R, Chirino AJ, Morag E, Bayer EA, Shoham Y, et al. Crystal structure of a bacterial family-III cellulose-binding domain: a general mechanism for attachment to cellulose. EMBO J. 1996;15(21):5739.

13. Georgelis N, Yennawar NH, Cosgrove DJ. Structural basis for entropy-driven cellulose binding by a

14. Morag E, Lapidot A, Govorko D, Lamed R, Wilchek M, Bayer EA, et al. Expression , Purification , and Characterization of the Cellulose- Binding Domain of the Scaffoldin Subunit from the Cellulosome of Clostridium thermocellum. Appl Environ Microbiol. 1995;61(5):1980-6.

15. Voutilainen S, Paananen A, Lille M, Linder M. Modular Protein Architectures for $\mathrm{pH}$-Dependent

16. Arola S, Linder MB. Binding properties of single and double cellulose binding modules reveal

17. Mohammadi P, Aranko AS, Lemetti L, Cenev Z, Zhou Q, Virtanen S, et al. Phase transitions as intermediate steps in the formation of molecularly engineered protein fibers. Commun Biol.

18. Lemetti L, Hirvonen S, Fedorov D, Batys P, Sammalkorpi M, Tenhu H, et al. Molecular crowding facilitates assembly of spidroin-like proteins through phase separation. Eur Polym J. 
Spectroscopy. Biochemistry. 1995;34(21):6993-7009.

419 20. Rowe AJ. Ultra-weak reversible protein - protein interactions. Methods. 2011;54(1):157-66.

420 21. Demeler B, Brookes E, Wang R, Schirf V, Kim CA. Characterization of reversible associations by 421 sedimentation velocity with ultrascan. Macromol Biosci. 2010;10(7):775-82.

422 22. Demeler B. A Comprehensive Data Analysis Software Package for Analytical Ultracentrifugation Experiments. In: Modern Analytical Ultracentrifugation Techniques, Royal Society of Chemistry. 2005. p. 210-29.

23. Brookes E, Demeler B. Genetic Algorithm Optimization for Obtaining Accurate Molecular Weight 2006;131:33-40.

24. Brookes EH, Demeler B. Parsimonious regularization using genetic algorithms applied to the analysis of analytical ultracentrifugation experiments. Proc 9th Annu Conf Genet Evol Comput. 2007;361-8.

25. Demeler B, Van Holde KE. Sedimentation velocity analysis of highly heterogeneous systems. Anal

26. Brookes E, Demeler B, Rosano C, Rocco M. The implementation of SOMO ( SOlution MOdeller ) in the UltraScan analytical ultracentrifugation data analysis suite : enhanced capabilities allow the

27. Kang E, Mansfield ML, Douglas JF. Numerical path integration technique for the calculation of reliable hydrodynamic modeling of virtually any kind of biomacromolecule. Eur Biophys J. transport properties of proteins. Phys Rev E. 2004;1-11.

28. Mansfield ML, Douglas JF. Improved path integration method for estimating the intrinsic viscosity

441 29. Juba D, Audus DJ, Mascagni M, Douglas JF, Keyrouz W. ZENO : Software for calculating hydrodynamic, electrical, and shape properties of polymer and particle suspensions. Journalof Res Natl Instituteof Stand andTechnology. 2017;122(20):1-2. 
31. Berendsen HJC, van der Spoel D, van Drunen R. GROMACS: A message-passing parallel molecular dynamics implementation. Comput Phys Commun. 1995;91(1-3):43-56.

32. Juréus A, Langel Ü. GROMACS 3.0: a package for molecular simulation and trajectory analysis. Acta Chim Slov. 1996;43(1):51-60.

33. Essmann U, Perera L, Berkowitz ML, Darden T, Lee H, Pedersen LG. A smooth particle mesh Ewald method. J Chem Phys. 1995;103(19):8577-93.

34. Best RB, Zheng W, Mittal J. Balanced protein-water interactions improve properties of disordered proteins and non-specific protein association. J Chem Theory Comput. 2014;10(11):5113-24.

35. Abascal JLF, Vega C. A general purpose model for the condensed phases of water : TIP4P / 2005. J Chem Phys. 2005;234505.

36. Bussi G, Donadio D, Parrinello M. Canonical sampling through velocity rescaling. J Chem Phys. 2007;126(1).

37. Parrinello $\mathrm{M}$, Rahman A. Polymorphic transitions in single crystals: A new molecular dynamics method. J Appl Phys. 1981;52(12):7182-90.

38. Humphrey W, Dalke A, Schulten K. VMD : Visual Molecular Dynamics. 1996;7855(December 1995):33-8.

39. Mao X, Ren Z, Parker GN, Sondermann H, Pastorello MA, Wang W, et al. Structural bases of unphosphorylated STAT1 association and receptor binding. Mol Cell. 2005;17(6):761-71.

40. Zheng J, Fage CD, Demeler B, Ho DW, Keatinge-clay AT. The Missing Linker: A Dimerization Motif Located within Polyketide Synthase Modules. 2013;

41. Silkowski H, Davis SJ, Barclay AN, Rowe AJ, Harding SE, Byron O. Characterisation of the low affinity interaction between rat cell adhesion molecules CD2 and CD48 by analytical ultracentrifugation. 1997;58:455-62. 


\section{Supporting information}

3 Analyzing the weak dimerization of a cellulose binding module by sedimentation velocity

4 experiments

Dmitrii Fedorov ${ }^{1}$, Piotr Batys ${ }^{1,2}$, Maria Sammalkorpi ${ }^{1,3}$, and Markus B. Linder ${ }^{1 *}$

$8{ }^{1}$ Department of Bioproducts and Biosystems, School of Chemical Engineering, Aalto

9 University, Espoo, Finland

$10{ }^{2}$ Jerzy Haber Institute of Catalysis and Surface Chemistry, Polish Academy of Sciences,

11 Krakow, Poland

$12{ }^{3}$ Department of Chemistry and Material Science, School of Chemical Engineering, Aalto

13 University, Espoo, Finland

14 *Corresponding author:

15 E-mail: markus.linder@aalto.fi

\section{Genetic algorithm (GA)}

18 GA analysis is based on previously obtained 2DSA results. As an initialization step, bucket

19 forms around each species. The width and the height of the bucket are automatically determined.

20 We used autoassignment to define solute bins, however it is possible to assign bins and insert the 21 parameters manually. Parameters of GA analysis were:

\begin{tabular}{|c|c|c|c|c|c|c|}
\hline Demes & Population size & Generations & $\begin{array}{l}\text { Crossover } \\
\text { percent }\end{array}$ & $\begin{array}{l}\text { Mutation } \\
\text { percent }\end{array}$ & Plague Percent & Elitism \\
\hline 1 & 200 & 100 & 50 & 50 & 4 & 2 \\
\hline $\begin{array}{l}\text { Migration } \\
\text { rate }\end{array}$ & Regularization & Random seed & $\begin{array}{l}\text { Simulation } \\
\text { points }\end{array}$ & $\begin{array}{l}\text { Band } \\
\text { loading } \\
\text { volume }\end{array}$ & $\begin{array}{l}\text { Concentration } \\
\text { threshold }\end{array}$ & S grid \\
\hline 3 & 5 & 0 & 200 & 0.015 & 0.00001 & 100 \\
\hline $\mathrm{K}$ grid & Mutate sigma & Mutate $\mathrm{s}$ & Mutate k & $\begin{array}{l}\text { Mutate } \mathrm{s} \\
\text { and } \mathrm{k}\end{array}$ & & \\
\hline 100 & 0 & 20 & 20 & 20 & & \\
\hline
\end{tabular}




\section{Genetic algorithm Monte Carlo (GAMC)}

25 GAMC is based on the GA results. At this point we do the same steps as for GA and use the 26 same parameters, only difference is that amount of Monte Carlo iterations equal to 64 . We used 27 parallel processing with 8 nodes to perform this type of analysis.

van Holde-Weischet (vHW) analysis

van Holde-Weischet distribution plots were obtained by using the following parameters:

\begin{tabular}{|l|l|l|l|l|}
\hline $\begin{array}{l}\text { Back diffusion } \\
\text { tolerance }\end{array}$ & Divisions & Data smoothing & \% of boundary & $\begin{array}{l}\text { Boundary position } \\
{[\%]}\end{array}$ \\
\hline 0.201 & 50 & 30 & 90 & 5 \\
\hline
\end{tabular}

31 In addition, about 10 - 15 first scans were deleted to decrease influence of instability in the

32 beginning of the experiment on distribution.

34 Discrete model genetic algorithm (DMGA)

35 The first step to perform DMGA is making the model and defining constrains.

36 The model consisted of two components: monomer and dimer, with the following parameters:

\begin{tabular}{|l|l|l|l|l|l|l|}
\hline & $\begin{array}{l}\text { Partial } \\
\text { spec. } \\
\text { volume } \\
{[\mathrm{mL} / \mathrm{g}]}\end{array}$ & $\begin{array}{l}\text { Extinction, OD } \\
{[\mathrm{mol} \cdot \mathrm{cm}]}\end{array}$ & Signal conc. & $\begin{array}{l}\text { Molecular } \\
\text { weight }\end{array}$ & $\begin{array}{l}\text { Frictional } \\
\text { ratio }\end{array}$ & $\begin{array}{l}\text { Conc. } \\
\text { dependency }\end{array}$ \\
\hline Monomer & 0.7148 & 35410 & 1.3436 & 18470 & 1.25 & 0 \\
\hline Dimer & 0.7148 & 70820 & 0 & 36940 & 1.40 & 0 \\
\hline
\end{tabular}

37 The signal concentration is the total concentration taken from final step of 2DSA.

38 DMGA Parameter constrains also were defined for monomer and dimer as follows:

\begin{tabular}{|l|l|l|l|l|l|l|}
\hline Demes & Population size & Generations & $\begin{array}{l}\text { Crossover } \\
\text { percent }\end{array}$ & $\begin{array}{l}\text { Mutation } \\
\text { percent }\end{array}$ & Plague Percent & Elitism \\
\hline 1 & 200 & 100 & 50 & 50 & 4 & 2 \\
\hline $\begin{array}{l}\text { Migration } \\
\text { rate }\end{array}$ & Random seed & $\begin{array}{l}\text { Simulation } \\
\text { points }\end{array}$ & $\begin{array}{l}\text { Band } \\
\text { loading } \\
\text { volume }\end{array}$ & $\begin{array}{l}\text { Constraints } \\
\text { parameter } \\
\text { grid }\end{array}$ & Mutate sigma & \\
\hline 3 & 0 & 200 & 0.015 & 1000 & 0 & \\
\hline
\end{tabular}

